\title{
ASSESSMENT OF THE GPC CONTROL QUALITY USING NON-GAUSSIAN STATISTICAL MEASURES
}

\author{
PAWEŁ D. DOMAŃSKI ${ }^{a}$, MACIEJ ŁAWRYŃCZUK $^{a, *}$ \\ ${ }^{a}$ Institute of Control and Computation Engineering, Faculty of Electronics and Information Technology \\ Warsaw University of Technology, ul. Nowowiejska 15/19, 00-665 Warsaw, Poland \\ e-mail: \{P.Domanski, M. Lawrynczuk\}@ia.pw.edu.p1
}

\begin{abstract}
This paper presents an alternative approach to the task of control performance assessment. Various statistical measures based on Gaussian and non-Gaussian distribution functions are evaluated. The analysis starts with the review of control error histograms followed by their statistical analysis using probability distribution functions. Simulation results obtained for a control system with the generalized predictive controller algorithm are considered. The proposed approach using Cauchy and Lévy $\alpha$-stable distributions shows robustness against disturbances and enables effective control loop quality evaluation. Tests of the predictive algorithm prove its ability to detect the impact of the main controller parameters, such as the model gain, the dynamics or the prediction horizon.
\end{abstract}

Keywords: control performance assessment, GPC control, non-Gaussian PDF, Cauchy PDF, Lévy $\alpha$-stable PDF.

\section{Introduction}

The presented work integrates different research areas: model predictive control (MPC) (Camacho and Bordons, 1999; Maciejowski, 2002; Tatjewski, 2007), control performance assessment (CPA) (Jelali, 2006) and statistics (Eisenhart, 2006). A rationale originates from practical aspects of advanced process control (APC) implementations. Good control performance plays crucial role in achieving benefits by any installation (Ordys et al., 2007). An improperly chosen control philosophy or a poor tuning deteriorate overall process performance. Moreover, industrial processes are mostly time varying complex systems. The results should not only be reached but also sustained. On-line performance monitoring and diagnostic start to play increasingly important role and become an inevitable element of good practice. This effect is even more visible in process optimization (PO) and APC (Smuts and Hussey, 2011) solutions, where regulation is more sensitive due to the operation close to constraints. Profound knowledge is needed for proper design, implementation and tuning.

Contemporary industrial control systems rely mainly on proportional-integral-derivative (PID) regulation. However, the improvements achievable with the PID

* Corresponding author algorithm are limited. APC techniques gain common acceptance. Advanced MPC algorithms compute the control signal on the basis of an embedded process model. While the model supports controller with prediction, optimization is used to calculate control rule minimizing costs and satisfying constraints. The MPC approach is very flexible, e.g., it makes it possible to control processes described by both linear (Tatjewski, 2014) and nonlinear models (Ławryńczuk, 2015; Tatjewski, 2007), may use on-line set-point optimization (Tatjewski, 2010) and may incorporate fault tolerant methods (Yang and Maciejowski, 2015). Generalized predictive control (GPC) is one of MPC algorithms. It was introduced by Clarke et al. (1987a) with several further extensions (Clarke et al., 1987b) and specific analyses (Clarke and Mohtadi, 1989). Although the algorithm is well established and there are a lot if reported successful implementations, its tuning and performance assessment is a challenging task. The selection of this algorithm is brought about by the fact that the parameters of the internal model, like gain, delay or time constant have a clear engineering meaning and are thus easily understood and interpreted by the control engineer during the assessment procedure.

The subject of CPA has been investigated for several years. It is important, as a bulk of process control 
loops perform poorly. $60 \%$ of all SISO loops have poor tuning and even more of them (85\%) an improper control structure (Jelali, 2013). The research started in the 1960s, getting popularity in 1989 with the minimum variance (MinVar) index (Harris, 1989). A real development using varying approaches, mostly MinVar, started and continues.

Mathematicians offer many different statistical approaches for data analysis. The Gaussian approach with normal probability distribution functions (PDFs) is the most popular one in control engineering. However, there are a lot of other distributions established in other contexts and offering attractive interpretations. Observation of control properties in many industrial examples (Domański, 2015) shows that the reality is not uniform. Controller variables and disturbances often cannot be rationally explained with standard linear and Gaussian approximations.

This paper tries to restore attractive features of other methods. Various statistical factors of non-Gaussian distributions are confronted and compared with the standard approach using the Gaussian normal distribution. Several different relations are analyzed, such as the impact of the GPC control horizon and misfits of gain, delay and time constant in the controller embedded model. Undisturbed and disturbed scenarios are considered to evaluate the robustness of selected indexes.

The proposed and evaluated methods show effectiveness against standard Gaussian factors. They are able to detect optimal values for main parameters of the GPC algorithm, like the internal model gain and dynamics or the prediction horizon. Proper detection is able also in the case of strong disturbances added into the loop.

Our analysis starts with the presentation of used algorithms and methods. The GPC algorithm is presented in Section 2, while Section 3 covers CPA approaches and evaluated indexes with a detailed description of statistical measures in Section 3.3). Main part of the paper includes data analysis for several simulation scenarios of a GPC loop (Section 4). The paper concludes in Section 5 with the discussion of results and open issues to be addressed in further research.

\section{Generalized predictive control}

2.1. Predictive control formulation. Let the input of the process (MV manipulated variable) be denoted by $u$ and the output of the process (CV controlled variable) by $y$. In contrast to the classical PID controller, which calculates at the current discrete sampling instant $k$ only the value of the manipulated variable for the current instant, i.e., $u(k)$, in MPC algorithms (Tatjewski, 2007) at each consecutive sampling instant $k$ a whole set of future control increments is calculated

$$
\triangle \boldsymbol{u}(k)=\left[\triangle u(k \mid k) \ldots \triangle u\left(k+N_{\mathrm{u}}-1 \mid k\right)\right]^{\mathrm{T}} .
$$

The number of decisions variables is determined by the control horizon, $N_{\mathrm{u}}$, and the increments are defined by

$$
\begin{aligned}
& \triangle u(k+p \mid k) \\
& \quad= \begin{cases}u(k \mid k)-u(k-1) & \text { if } p=0, \\
u(k+p \mid k)-u(k+p-1 \mid k) & \text { if } p \geq 1 .\end{cases}
\end{aligned}
$$

It is assumed that $\triangle u(k+p \mid k)=0$ for $p \geq N_{\mathrm{u}}$, i.e., $u(k+p \mid k)=u\left(k+N_{\mathrm{u}}-1 \mid k\right)$ for $p \geq N_{\mathrm{u}}$. The increments in the future values of the manipulated variable (1) are calculated from an optimization problem, in which the predicted control quality is maximized and the constraints are taken into account. Typically, the predicted control quality is defined as forecast control errors, i.e., the differences between the set-point, $y^{\mathrm{sp}}(k+p \mid k)$, and the predicted process output, $\hat{y}(k+p \mid k)$, for $p=N_{1}, \ldots, N_{2}$, where $N_{1}$ and $N_{2}$ determine the beginning and the end of the prediction horizon, respectively. Assuming that there are constraints imposed on the range of the MV defined by the real numbers $u^{\min }, u^{\max }$, the constraints imposed on the rate of change of the MV defined by $\triangle u^{\min }, \triangle u^{\max }$ and the constraints imposed on the range of the predicted controlled variable defined by $y^{\min }, y^{\max }$, the general MPC optimization problem is

$$
\begin{gathered}
\min _{\triangle \boldsymbol{u}(k)}\left\{J(k)=\sum_{p=N_{1}}^{N_{2}}\left(y^{\mathrm{ref}}(k+p \mid k)-\hat{y}(k+p \mid k)\right)^{2}\right. \\
\left.\quad+\sum_{p=0}^{N_{\mathrm{u}}-1} \lambda(\Delta u(k+p \mid k))^{2}\right\}
\end{gathered}
$$

subject to

$$
\begin{aligned}
u^{\min } & \leq u(k+p \mid k) \leq u^{\max }, & & p=0, \ldots, N_{\mathrm{u}}-1, \\
\triangle u^{\min } & \leq \triangle u(k+p \mid k) \leq \Delta u^{\max }, & & p=0, \ldots, N_{\mathrm{u}}-1, \\
y^{\min } & \leq \hat{y}(k+p \mid k) \leq y^{\max }, & & p=N_{1}, \ldots, N_{2} .
\end{aligned}
$$

The optimization problem (2) is solved on-line. As a result, future control increments (1) are calculated, but only the first element of the sequence is actually applied to the process, i.e., $u(k)=\Delta u(k \mid k)+u(k-1)$. At the next sampling instant, $k+1$, the prediction is shifted one step forward and the whole procedure is repeated. The second part of the minimized cost-function $J(k)$ is a penalty term $(\lambda>0$ is a weighting coefficient), which may be used to slow down the trajectories, but primarily it is used to obtain good numerical properties of the MPC optimization problem. If the MPC optimization problem takes into account the constraints imposed on the predicted process output, it may be necessary to 
enforce the existence of the feasible set, e.g., by softening such constraints, when the set of possible solutions to the MPC optimization problem is empty (Maciejowski, 2002; Tatjewski, 2007). For further description, the MPC optimization problem (2) is expressed compactly in a vector-matrix notation

$$
\min _{\triangle \boldsymbol{u}(k)}\left\{\left\|\boldsymbol{y}^{\mathrm{sp}}(k)-\hat{\boldsymbol{y}}(k)\right\|^{2}+\|\Delta \boldsymbol{u}(k)\|_{\boldsymbol{\Lambda}}^{2}\right\},
$$

subject to

$$
\begin{aligned}
\boldsymbol{u}^{\min } & \leq \boldsymbol{J} \triangle \boldsymbol{u}(k)+\boldsymbol{u}(k-1) \leq \boldsymbol{u}^{\max }, \\
-\triangle \boldsymbol{u}^{\max } & \leq \Delta \boldsymbol{u}(k) \leq \Delta \boldsymbol{u}^{\max } \\
\boldsymbol{y}^{\min } & \leq \hat{\boldsymbol{y}}(k) \triangle \boldsymbol{u}(k) \leq \boldsymbol{y}^{\max } .
\end{aligned}
$$

where the norms are defined as $\|\boldsymbol{x}\|^{2}=\boldsymbol{x}^{\mathrm{T}} \boldsymbol{x}$ and $\|\boldsymbol{x}\|_{\boldsymbol{A}}^{2}=\boldsymbol{x}^{\mathrm{T}} \boldsymbol{A} \boldsymbol{x}$, the set-point trajectory vector $\boldsymbol{y}^{\mathrm{sp}}(k)=$ $\left[y^{\mathrm{sp}}\left(k+N_{1} \mid k\right) \ldots y^{\mathrm{sp}}\left(k+N_{2} \mid k\right)\right]^{\mathrm{T}}, \quad$ the predicted trajectory vector $\hat{\boldsymbol{y}}(k)=\left[\hat{y}\left(k+N_{1} \mid k\right) \ldots \hat{y}\left(k+N_{2} \mid k\right)\right]^{\mathrm{T}}$ and the vectors which define the output constraints, i.e., $\boldsymbol{y}^{\min }=\left[y^{\min } \ldots y^{\min }\right]^{\mathrm{T}}, \boldsymbol{y}^{\max }=\left[y^{\max } \ldots y^{\max }\right]^{\mathrm{T}}$, are of length $N_{2}-N_{1}+1$. The vectors which define the input constraints, i.e., $\boldsymbol{u}^{\min }=\left[u^{\min } \ldots u^{\min }\right]^{\mathrm{T}}, \boldsymbol{u}^{\max }=$ $\left[u^{\max } \ldots u^{\max }\right]^{\mathrm{T}}, \Delta \boldsymbol{u}^{\max }=\left[\Delta u^{\max } \ldots \Delta u^{\max }\right]^{\mathrm{T}}$ and the vector $\boldsymbol{u}(k-1)=[u(k-1) \ldots u(k-1)]^{\mathrm{T}}$ are of length $N_{\mathrm{u}}$, the matrices $\boldsymbol{\Lambda}=\operatorname{diag}(\lambda, \ldots, \lambda)$ and

$$
\boldsymbol{J}=\left[\begin{array}{ccccc}
1 & 0 & 0 & \ldots & 0 \\
1 & 1 & 0 & \ldots & 0 \\
\vdots & \vdots & \vdots & \ddots & \vdots \\
1 & 1 & 1 & \ldots & 1
\end{array}\right]
$$

is an $N_{\mathrm{u}} \times N_{\mathrm{u}}$ matrix.

\subsection{Generalized predictive control implementation.} In all MPC algorithms a dynamic model of the controlled process is used to predict the future values of the output variable, $\hat{y}(k+p \mid k)$, over the prediction horizon, i.e., for $p=N_{1}, \ldots, N_{2}$. In the GPC algorithm the model of the process has the form of the discrete difference equation

$$
\boldsymbol{A}\left(q^{-1}\right) y(k)=\boldsymbol{B}\left(q^{-1}\right) u(k-1)+\boldsymbol{C}\left(q^{-1}\right) \frac{\epsilon(k)}{\triangle},
$$

where the polynomials in the $q^{-1}$ shift operator are

$$
\begin{aligned}
& \boldsymbol{A}\left(q^{-1}\right)=1+a_{1} q^{-1}+\ldots+a_{n_{\mathrm{A}}} q^{-n_{\mathrm{A}}}, \\
& \boldsymbol{B}\left(q^{-1}\right)=b_{1} q^{-1}+\ldots+b_{n_{\mathrm{B}}} q^{-n_{\mathrm{B}}}, \\
& \boldsymbol{C}\left(q^{-1}\right)=1+c_{1} q^{-1}+\ldots+c_{n_{\mathrm{C}}} q^{-n_{\mathrm{C}}} .
\end{aligned}
$$

Here $\epsilon(k)$ denotes the white noise vector with zero mean and $\triangle=1-q^{-1}$ stands for the backward-difference operator $(1 / \triangle$ means integration). The model (5) may be called auto-regressive integrated moving average with exogenous input (ARIMAX) or controlled auto-regressive integrated moving average (CARIMA) (Tatjewski, 2007). Assuming that the process is affected by an integrated white noise $\left(\boldsymbol{C}\left(q^{-1}\right)=1\right)$ the model (5) becomes

$$
\boldsymbol{A}\left(q^{-1}\right) y(k)=\boldsymbol{B}\left(q^{-1}\right) u(k-1)+\frac{\epsilon(k)}{\triangle} .
$$

The above model is often used in practical implementations of the GPC algorithm since the assumption that $\boldsymbol{C}\left(q^{-1}\right)=1$ enables easy derivation of prediction equations when compared with the general case $\boldsymbol{C}\left(q^{-1}\right) \neq 1$, i.e., when the integrated noise is colored. The model (9) is used to derive the prediction equation (Clarke et al., 1987a)

$$
\hat{\boldsymbol{y}}(k)=\boldsymbol{G} \triangle \boldsymbol{u}(k)+\boldsymbol{y}^{0}(k),
$$

where the so-called dynamic matrix $\boldsymbol{G}$ (calculated once, off-line) of dimensionality $\left(N_{2}-N_{1}+1\right) \times N_{\mathrm{u}}$ consists of the step-response coefficients of the model (9) and the free trajectory $\boldsymbol{y}^{0}(k)=\left[y^{0}\left(k+N_{1} \mid k\right) \ldots y^{0}\left(k+N_{2} \mid k\right)\right]^{\mathrm{T}}$ (calculated at each sampling instant $k$ ) is evaluated from

$$
\boldsymbol{y}^{0}(k)=\boldsymbol{F} \boldsymbol{y}^{\mathrm{PG}}(k)+\boldsymbol{G}^{\mathrm{PG}} \triangle \boldsymbol{u}^{\mathrm{PG}}(k),
$$

where the vectors $\boldsymbol{y}^{\mathrm{PG}}(k)=\left[y(k) \ldots y\left(k-n_{\mathrm{A}}\right)\right]^{\mathrm{T}}$ and $\boldsymbol{u}^{\mathrm{PG}}(k)=\left[\Delta u(k-1) \ldots \Delta u\left(k-n_{\mathrm{B}}\right)\right]^{\mathrm{T}}$ are of length $n_{\mathrm{A}}+1$ and $n_{\mathrm{B}}$, respectively, and the matrices $\boldsymbol{F}$ and $\boldsymbol{G}^{\mathrm{PG}}$, of dimensionalities $\left(N_{2}-N_{1}+1\right) \times\left(n_{\mathrm{A}}+1\right)$ and $\left(N_{2}-N_{1}+1\right) \times n_{\mathrm{B}}$ are calculated from the model equation (9) by solving Diophantine equations (Clarke et al., 1987a; Tatjewski, 2007). From Eqns. (10) and (11), the GPC prediction equation is derived

$$
\hat{\boldsymbol{y}}(k)=\boldsymbol{G} \triangle \boldsymbol{u}(k)+\boldsymbol{F} \boldsymbol{y}^{\mathrm{PG}}(k)+\boldsymbol{G}^{\mathrm{PG}} \triangle \boldsymbol{u}^{\mathrm{PG}}(k) .
$$

It is necessary to point out that the future predictions of the $\mathrm{CV}$ are linear functions of the calculated decision vector $\triangle \boldsymbol{u}(k)$ according to the GPC prediction equation (12) and the free trajectory depends only on the past. Using (12), from the general MPC optimization problem (3), we obtain the GPC minimization task

$$
\begin{aligned}
\min _{\triangle \boldsymbol{u}(k)}\left\{\| \boldsymbol{y}^{\mathrm{sp}}(k)-\boldsymbol{G} \triangle \boldsymbol{u}(k)-\boldsymbol{F} \boldsymbol{y}^{\mathrm{PG}}(k)\right. \\
\left.\quad-\boldsymbol{G}^{\mathrm{PG}} \triangle \boldsymbol{u}^{\mathrm{PG}}(k)\left\|^{2}+\right\| \Delta \boldsymbol{u}(k) \|_{\boldsymbol{\Lambda}}^{2}\right\},
\end{aligned}
$$

subject to

$$
\begin{aligned}
\boldsymbol{u}^{\min } \leq & \boldsymbol{J} \triangle \boldsymbol{u}(k)+\boldsymbol{u}(k-1) \leq \boldsymbol{u}^{\max }, \\
-\triangle \boldsymbol{u}^{\max } \leq & \triangle \boldsymbol{u}(k) \leq \triangle \boldsymbol{u}^{\max } \\
\boldsymbol{y}^{\min } \leq & \boldsymbol{G} \triangle \boldsymbol{u}(k)+\boldsymbol{F} \boldsymbol{y}^{\mathrm{PG}}(k) \\
& +\boldsymbol{G}^{\mathrm{PG}} \triangle \boldsymbol{u}^{\mathrm{PG}}(k) \triangle \boldsymbol{u}(k) \leq \boldsymbol{y}^{\max } .
\end{aligned}
$$

As the prediction equation (12) is linear in terms of the vector $\triangle \boldsymbol{u}(k)$, the obtained optimization problem (13) is of the quadratic programming (QP) type, i.e., the cost function is quadratic and all constraints are linear. 


\section{Control performance assessment}

Control engineers use various approaches to assess quality. Their methods originate from personal experience. Benchmarking was introduced for business processes in the late 1970s (Cross and Iqbal, 1995) and the first reported loop performance assessment was done by Åström for a paper machine in 1967 (Åström, 1967). The development of CPA tools pursued. It gained big attention in 1989 (Harris, 1989) with the introduction of the performance index based on the MinVar approach. Now research covers many different aspects of control, such as nonlinear behavior (Horch and Isaksson, 1998), large scale systems (Paulonis and Cox, 2003), predictive control (Schäfer and Cinar, 2004), SISO (Hugo, 2006) and MIMO structures (Zhuo, 2009), valve stiction (Choudhury et al., 2008), oscillations (Srinivasan and Rengaswamy, 2012) and many others. Industrial approaches are mostly in the form of financial or technical KPIs (Thornhill and Shah, 2005). Nowadays we observe approaches unequal to the above ones using a nonlinear data analysis approach. They are not based on Gaussian assumptions (Domański, 2016).

Concluding, there exist many CPA approaches, methodologies and algorithms. We may distinguish five main classes of methods:

1. time-domain indexes based on the step test response, such as delay, undershoot, rising, maximum and settling time, overshoot, peak amplitude, etc.;

2. time-domain approaches using control error signal, as for instance integral indexes, i.e., the mean integral square error (MISE), the integral of absolute error (IAE), the amplitude index (AMP), etc.;

3. MinVar indexes originating from Harris' approach;

4. statistical measures based on signal (control error, output) time series analysis with different PDFs factors, such as the standard deviation, scaling, skewness, position, etc.;

5. others such as wavelet, fractal or entropy measures.

This paper will focus on a comparison of standard approaches and non-Gaussian statistical measures.

3.1. Time domain CPA methods. Traditional step test measures, although very informative, have a limited industrial applicability. Industry hardly agrees to disturb a normal operation with testing experiments. On the other hand, the integral indexes are extremely popular and widely used. For further analysis three of them will be evaluated and compared: the MISE, IAE and AMP (Spinner et al., 2014).

As the difference between the MISE and IAE is rather minor, they are often used interchangeably without a deeper reflection. However, in the literature we may find some interesting comments. Seborg et al. (2010) showed that tuning to minimize the MISE punishes large deviations from the set-point, but also produces aggressive actions. On the other hand, Shinskey (2002) suggested that the IAE has the closest relationship to economic considerations. In the present analysis both will be calculated and compared.

3.2. Minimum variance CPA. The standard form of the MinVar index is calculated from

$$
H_{\text {index }}=\frac{\sigma_{\min V a r}^{2}}{\sigma_{y}^{2}}=\frac{\sum_{i=0}^{\tau-1} f_{i}^{2}}{\sum_{i=0}^{\infty} f_{i}^{2}} .
$$

where $f_{i}$ denotes the coefficients of the impulse response from the noise-to-output transfer function and they need to be estimated for instance with an ARMA model. Often the normalized version of Eqn. (14) is used,

$$
N_{\text {index }}=1-\frac{\sigma_{y}^{2}}{\sigma_{\min V a r}^{2}} .
$$

This index is very informative and constructive. But its evaluation may be practically limited by the assumption that the process structure, at least its delay, has to be known.

3.3. Statistical indexes. Statistical properties of the Gaussian normal distribution form a very popular set of KPIs. The mean value $x_{o}$ and the standard deviation $\sigma$ are commonly used. A non-zero mean value of the control error indicates whether the loop witnesses steady-state error. Furthermore, the standard deviation informs about the accuracy of set-point tracking. These measures are frequently supplemented with higher order statistics. Skewness

$$
\text { skewness }(x)=\frac{1}{N \sigma^{3}} \sum_{i=1}^{N}\left(x_{i}-\bar{x}\right)^{3},
$$

is a measure of PDF asymmetry. It informs about a positive or a negative bias from the mean value. For a symmetrical distribution it is equal to zero. If it is below zero, there are more negative values in data. While it is positive, we face the opposite situation. Kurtosis

$$
\text { kurtosis }(x)=\frac{1}{N \sigma^{4}} \sum_{i=1}^{N}\left(x_{i}-\bar{x}\right)^{4}-3
$$

is a shape descriptor. It measures data concentration. The higher kurtosis, the more scattered data are and the 


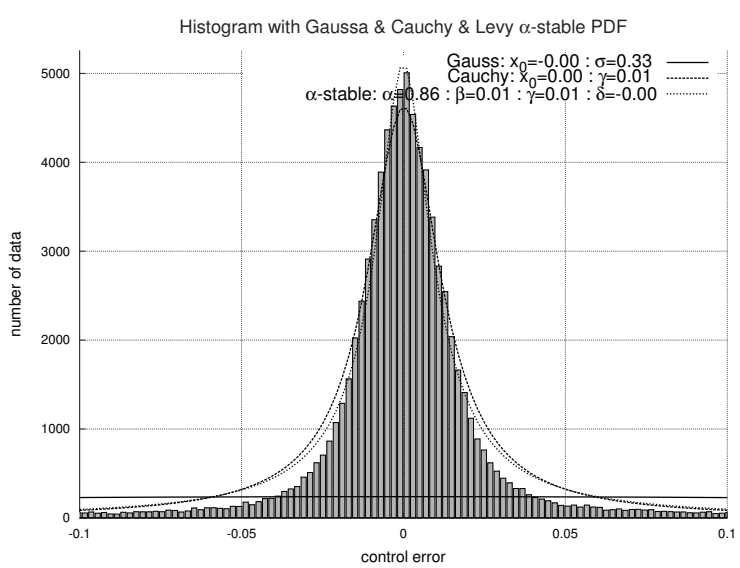

Fig. 1. Control error variable histogram with a fat tale shape best fitted with an $\alpha$-stable PDF.

distribution function shape is flatter. Small values result in a slender PDF.

We have to check whether or not variable properties are Gaussian. Such tests may be simply performed graphically through visual inspection of the histogram (see Fig. 10. We may calculate the mean square error between histogram and normal PDF fitted to data. Additionally, normality may be validated through specific tests, such as Kolmogorov-Smirnov (KS) normality test. Another approach is to check the normality hypothesis through skewness, kurtosis or $\alpha$ stability. Besides, one may use other tests: Smirnov-Cramer von Mises (Cramer, 1928), Anderson-Darling (Anderson and Darling, 1954), Shapiro-Wilk (Shapiro and Wilk, 1965), Lilliefors (Lilliefors, 1967).

A review of industrial loops (Domański, 2015) showed that only a minority $(\approx 6 \%)$ have normal properties. It was discovered that a majority have fat tails of a Lévy $\alpha$-stable $(>60 \%$ ) or a Cauchy distribution $(\approx 30 \%)$. This is probably caused by the complex nature of real industrial loops with non-linear and time varying cross coupling, nonlinearities, variable delays and frequent human interventions. It has been decided to evaluate the fitting of other probabilistic distribution functions to cover these aspects: Cauchy, Lévy $\alpha$-stable, Laplace, the unbounded Johnson function $\left(S_{U}\right)$ and the generalized extreme value (GEV).

3.3.1. Cauchy PDF. The Cauchy distribution is described by the following density function:

$$
P D F_{\delta, \gamma}(t)=\frac{1}{\pi \gamma}\left(\frac{\gamma^{2}}{(t-\delta)^{2}+\gamma^{2}}\right),
$$

where $\delta \in \mathbb{R}$ denotes a location and $\gamma>0$ is a scale factor.
3.3.2. $\alpha$-stable distributions. Another interesting alternative for a good fitting might be the Lévy $\alpha$-stable distribution characteristics equation

$$
P D F_{\alpha, \beta, \delta, \gamma}(t)=\exp \left\{i \delta t-|\gamma t|^{\alpha}(1-i \beta l(t))\right\},
$$

where

$$
l(t)= \begin{cases}\operatorname{sgn}(t) \tan \left(\frac{\pi \alpha}{2}\right) & \text { if } \alpha \neq 1 \\ -\operatorname{sgn}(t) \frac{2}{\pi} \ln |t| & \text { if } \alpha=1\end{cases}
$$

Here $0<\alpha \leq 2$ is called the stability index, $|\beta| \leq 1$ is skewness, $\delta \in \mathbb{R}$ is the location and $\gamma>0$ is a scale factor.

3.3.3. Laplace PDF. The Laplace distribution is sometimes called the double exponential distribution. It forms a function of differences between two independent variables with identical exponential distributions. Its probability density function is given by

$$
P D F_{\mu, b}=\frac{1}{2 b} e^{-\frac{|x-\mu|}{b}},
$$

where $\mu \in \mathbb{R}$ is a location and $b>0$ is a scale parameter.

3.3.4. Unbounded Johnson function $S_{U}$. The Johnson $S_{U}$ distribution is a four-parameter family of probability functions. It was proposed as a transformation of normal distribution with the PDF function given by

$$
\begin{aligned}
P D F_{\gamma, \xi, \delta, \lambda}= & \frac{\delta}{\lambda \sqrt{2 \pi}} \frac{1}{\sqrt{1+\left(\frac{x-\xi}{\lambda}\right)^{2}}} \\
& \times e^{-\frac{1}{2}\left(\gamma+\delta \sinh ^{-1}\left(\frac{x-\xi}{\lambda}\right)\right)^{2}},
\end{aligned}
$$

where $\gamma \in \mathbb{R}$ and $\delta>0$ are PDF shape parameters, $\xi \in \mathbb{R}$ is a distribution location and $\lambda>0$ is a scale factor.

3.3.5. Generalized extreme value distribution. The GEV distribution is a family of continuous PDFs developed within extreme value theory to combine properties of different distributions. Its distribution function is described by the following formula:

$$
P D F_{\mu, \sigma, \xi}=\frac{1}{\sigma} t(x)^{\xi+1} e^{-t(x)},
$$

where

$$
t(x)= \begin{cases}\left(1+\left(\frac{x-\mu}{\sigma}\right) \xi\right)^{-\frac{1}{\xi}} & \text { if } \xi \neq 0 \\ e^{-\frac{x-\mu}{\sigma}} & \text { if } \xi=0\end{cases}
$$

$\mu \in \mathbb{R}$ is a location, $\sigma>0$ a scale and $\xi \in \mathbb{R}$ a shape factor.

There are efficient algorithms (Borak et al., 2011) to fit the PDF to the histogram. In this paper 
the Cauchy parameter calculation uses maximum likelihood estimation (MLE) from the Octave successive quadratic programming solver (Axensten, 2006). Furthermore, $\alpha$-stable fitting applies the regression method (Koutrouvelis, 1980). Laplace and GEV estimation also uses MLE and the Johnson fitting is done with moments method (Hill et al., 1976).

\section{Simulations}

4.1. Simulation example. In order to demonstrate the usefulness of the discussed approach, the following continuous-time dynamic system is considered:

$$
G(s)=\frac{K}{\left(T_{1} s+1\right)\left(T_{2} s+1\right)} e^{-T_{0} s},
$$

where the nominal parameters are $K=2, T_{0}=4, T_{1}=$ $3, T_{2}=10$. Assuming the sampling period $0.5 \mathrm{sec}$., the discrete-time version of the transfer function $(23)$ is

$$
\left(1+a_{1} q^{-1}+a_{2} q^{-2}\right) y(k)=\left(b_{8} q^{-8}+b_{9}\right) u(k-1),
$$

where $a_{1}=-1.7977 \times 10^{-1}, b_{2}=8.0520 \times 10^{-1}, b_{8}=$ $7.7574 \times 10^{-3}, b_{9}=7.2169 \times 10^{-3}$ and the order of the model dynamics is defined by $n_{\mathrm{A}}=2, n_{\mathrm{B}}=9$. During GPC simulations, the constraints imposed on the range of the $\mathrm{MV}$ are taken into account, $u^{\min }=-1, u^{\max }=1$, as well as the constraints of the change rate: $\Delta u^{\mathrm{min}}=$ $-0.05, \triangle u^{\max }=0.05$.

After tuning it is found out that the control horizon should be $N_{\mathrm{u}}=3$, the prediction horizon should be defined by $N_{1}=9, N_{2}=25$, the penalty term should be $\lambda=0.5$. For the tuning of the GPC algorithm no plant-model mismatch is assumed, i.e., the model is perfect. The simulation control loop is also expanded with disturbances. A disturbance $z(t)$ with $\alpha$-stable PDF characteristics is added before the process, while Gaussian noise $d(t)$ is added at the process output.

Four scenarios are run to check how disturbances and the modeling mismatch affect control quality:

(Sc1) The real process gain value differs from that of the model used for GPC design, $K=2$. Seven different model gains are applied: $0.4,0.8,1.2, \mathbf{2 . 0}, 2.8,3.2$, 3.6.

(Sc2) The GPC applied prediction horizon length varies from the tuned one $N_{2}=25$. Six other lengths are also considered: $10,12,15,20,25,30,35$.

(Sc3) The real process delay value differs from that of the model used for GPC design, $T_{0}=8$. Nine different process delays are used: $4,5,6,7, \mathbf{8}, 9,10,11,12$.

(Sc4) The real value of the time constant, $T_{2}$, is different from the tuning model one $T_{2}=10$. Seven different values are checked: $0.5,1,5, \mathbf{1 0}, 15,20,40$.
Three different scenarios of disturbances are considered in addition to the above experiments. Two types of disturbances are considered. A Gaussian signal added at the process output is used to model measurement noise. A long-tail stochastic process modeled with a Lévy $\alpha$-stable probabilistic distribution function models main process disturbances. A review of many industrial control loops has shown that a majority of them were showing long-tail properties with the best function fitting by stable distributions (Domański, 2016). The other assumptions are as follows:

1. The process is not exposed to any disturbance.

2. The control loop is affected by additive input disturbances in the form of random values with a normal distribution and an amplitude of 0.008 .

3. The control loop is affected by additive input disturbances in the form of random values. It has a $\alpha$-stable distribution with an amplitude of 0.04 .

Additionally, the nominal GPC control loop is simulated with different noise characteristics, i.e.,

1. no disturbances,

2. three levels of normal noise (small, medium, large),

3. stability factors $\alpha=1.5,1.75,2$ of $\alpha$-stable distribution ( $\alpha=2$ means independent realizations).

For better visualization, two sample trends are added in Fig. 2.

Scenarios are used to answer the following questions:

(H0) Are the measures robust to disturbance properties? Can we evaluate loop quality despite disturbances?

(H1) Does the set-point have any impact on the results of the loop quality assessment?

(H2) Can we identify whether the GPC gain is appropriate?

(H3) Can we estimate if the GPC horizon is set properly?

(H4) Can we confirm whether the GPC delay is appropriate?

(H5) Can we say anything about the GPC embedded model dynamics (internal model time constant)?

Loop quality assessment is made with measures based on the control error trend (MISE, IAE, AMP), standard and normalized MinVar and statistical factors of Gaussian, Cauchy, $\alpha$-stable, Laplace, Johnson $S_{U}$ and GEV PDFs. 


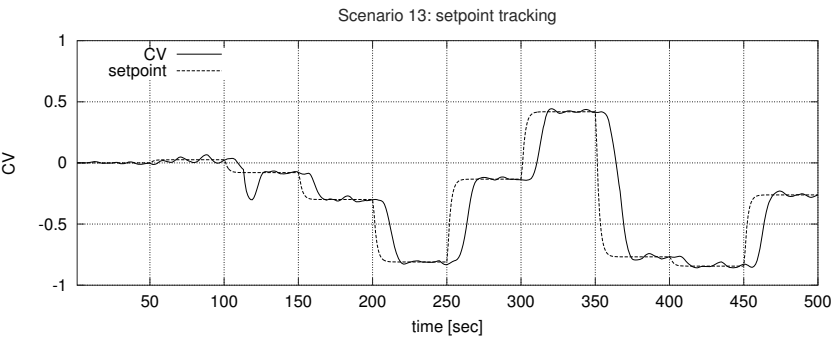

Scenario 13: MV trend

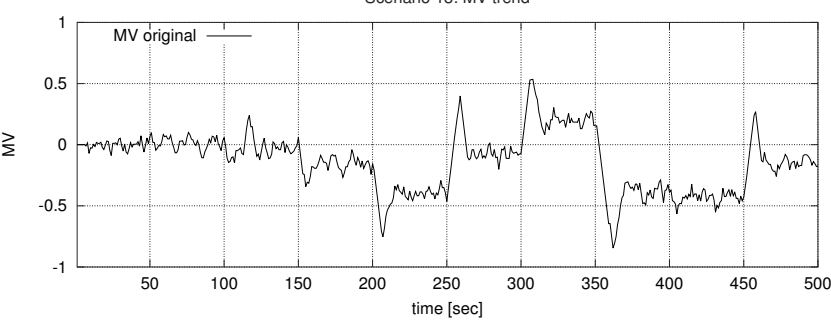

Fig. 2. Example time trends for a case with significant long-tail disturbance and ideal parameters.
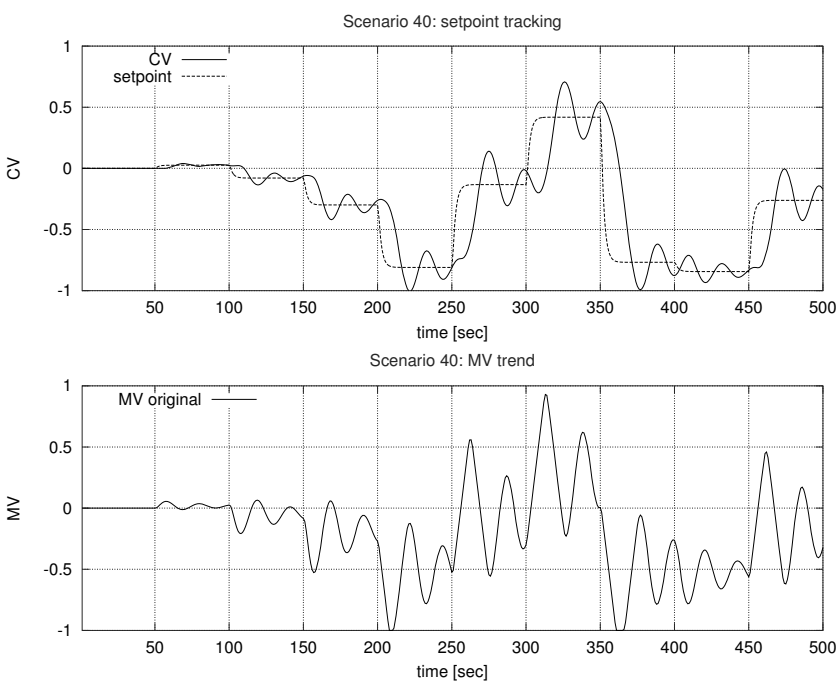

Fig. 3. Example time trends for a gain mismatch and a Gaussian disturbance.

4.2. Results. Presentation and discussion of the results is organized systematically according to the hypotheses $H 0, \ldots, H 5$. The indexes are grouped according to the role they play in distribution shaping. We may distinguish:

- Location factors visualize the average value of the distribution. In our case of control error, it shows how effectively the set-point is tracked by the process variable. Thus we expect the zero mean value for a good loop. Any non-zero number would reflect steady-state error or other loop problems, such as actuator nonlinearities or frequent manual interventions.

These are the following: Gauss $x_{o}$, Cauchy and Lévy

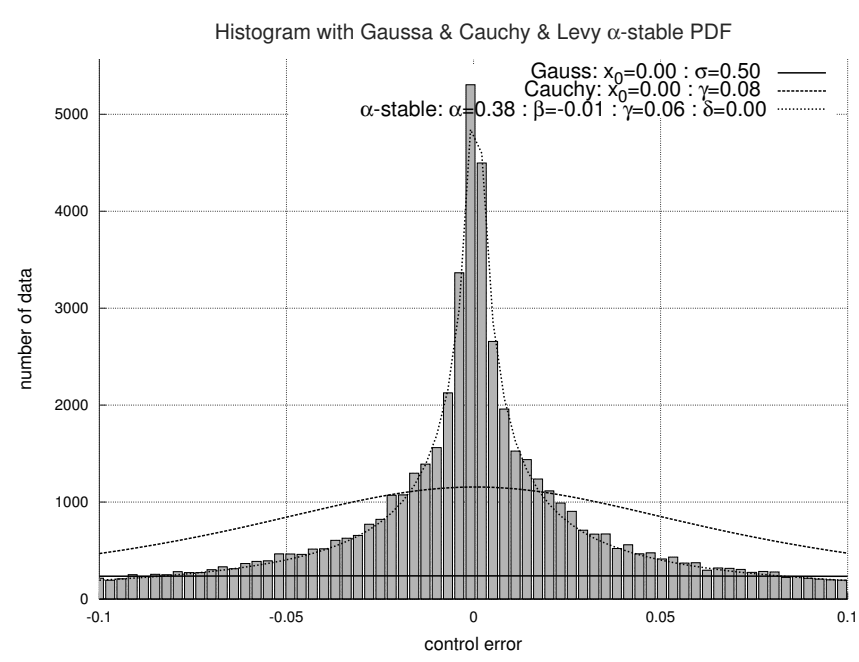

Fig. 4. Example of PDF fitting to a control error histogram for Gauss, Cauchy and Lévy.

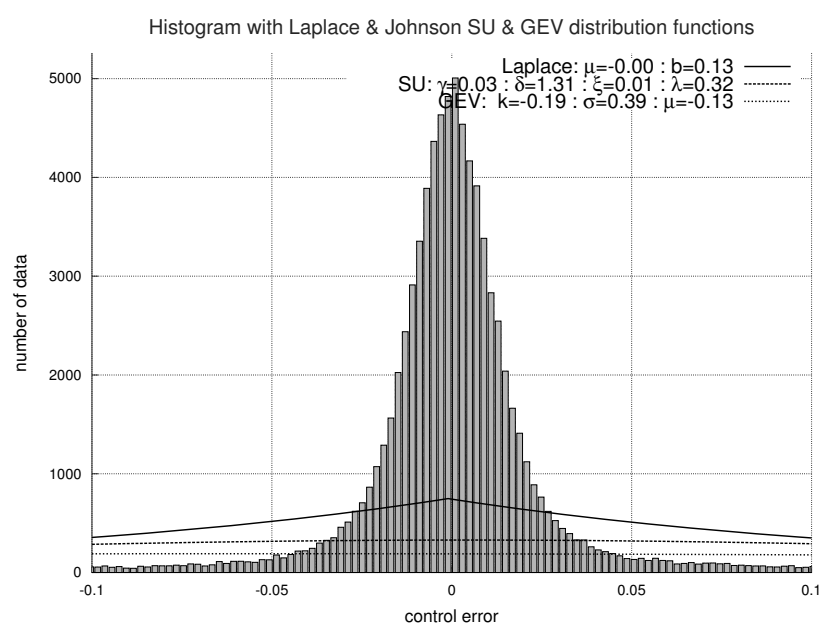

Fig. 5. Example of PDF fitting to a control error histogram for Laplace, $S_{U}$ and the GEV.

$\delta$, Laplace and GEV $\mu$ and $S_{U}$ factor $\xi$.

- Distribution scale parameters reflect data dispersion (variability). In our case we prefer that value to be as small as possible. Unfortunately, we hardly know its minimum (achievable) value and we have to base monitoring on the relative values. We may only see whether it is getting better or worse.

As for selected PDFs, we have Gauss $\sigma$, Cauchy and Lévy $\gamma$, Laplace $b$, GEV $\sigma$ and factor $\delta$ for $S_{U}$.

- For some of the distributions we have additional shape factors. They include information about skewness, slenderness or fat tails. In this group we 
count the Lévy $\alpha$ stability factor responsible for tails and the $\beta$ skewness parameter, GEV $\xi$ shape factor, $\gamma$ and $\delta$ for $S_{U}$ distribution. All the PDFs are fitted to control error histograms for all simulated scenarios. Example situations are sketched in Figs. 4 and 5. They show that histograms of simulated control error feature fat-tail properties. Additionally, it may be noticed that histogram properties are only reflected by a $\alpha$-stable distribution.

4.2.1. Location estimation. The results of 162 simulations with different GPC parameters and loop disturbance profiles are included in one file and a statistical comparison of the obtained results is performed. According to the zero steady-state error assumption, all should detect the value equal to zero. Unfortunately, $S_{U}$ and GEV parameters do not reflect exactly the statistical mean value. For that purpose, for both of them the mean value is calculated

$$
\text { mean }_{S U}=\xi-\lambda \exp \left(\frac{\delta^{-2}}{2}\right) \sinh \left(\frac{\gamma}{\delta}\right),
$$

and

$$
\operatorname{mean}_{G E V}=\mu-\frac{\sigma}{\xi}+\frac{\sigma}{\xi} \cdot \Gamma(1-\xi) .
$$

Table 1 shows position estimates with the mean, minimum, maximum and variance for each parameter. We see that only the Johnson $S_{U}$ distribution fails, while all the others properly estimate the control error mean within satisfactory limitations, despite loop disturbances. The position estimates are independent of other CPA measures. From that perspective there is no reason to compare them with other indexes.

The correlation table (without Johnson $S_{U}$ ) is presented as Table 2. High correlations with absolute values larger than 0.8 are highlighted. The main observation is that estimates keep different properties of the histogram and in the case of loop disturbances they may behave in an unlikely manner. Actually, only Laplace and Gauss are exactly the same.

4.2.2. Scaling estimates. All 162 simulations are analyzed. The assumption is that the scaling factors should reflect control error variability. However, similarly to the mean value analysis data for $S_{U}$ and the GEV, the distributions have to be recalculated as their standard deviations are

$$
\begin{aligned}
\operatorname{stdev}_{S U}= & \sqrt{\frac{\lambda^{2}}{2}\left(\exp \left(\delta^{-2}\right)-1\right)} \\
& \times \sqrt{\left(\exp \left(\delta^{-2}\right) \cosh \left(\frac{2 \gamma}{\delta}\right)+1\right)},
\end{aligned}
$$

and

$$
\operatorname{stdev}_{G E V}=\sqrt{\frac{\sigma^{2}}{\xi^{2}}\left(\Gamma(1-2 \xi)-\Gamma^{2}(1-\xi)\right)},
$$

respectively.

Table 3 includes the mean, the minimum, the maximum and the variance for each scaling factor. In that case, the interpretation is not so straightforward as in the previous case. Control error variability exists and should be detected. The main observation is that they behave with different schemes.

It is interesting to see how they are interconnected. Their cross correlations are calculated and shown in Table 4 for that purpose. We see that both Cauchy and Lévy $\gamma$ parameters address very similar features. It is interesting to see that the Laplace scaling factor is also similar. We notice that $S_{U}$ and GEV distributions most probably reflect unlikely phenomena. Further, they will be confronted with other quality indexes (Section 4.2.6).

4.2.3. Shaping parameters. Finally, a similar comparison is made for shaping parameters (Table 5). The mean, minimum, maximum and variance for each of the parameter are calculated. We notice that these parameters are varying in a wide range. Kurtosis changes from a value close to zero up to large numbers. Also the Gauss skewness factor is characterized by the mean close to zero (as expected) varying from a negative to a positive number. These changes are connected with the noise and the disturbance character equivalent to performed scenarios.

We also see that the Lévy stability parameter $\alpha$ varies between 0 and 2, but without reaching 2 . The estimates cover the whole parameter feasible range. Here $\alpha=2$ means an uncorrelated stochastic process and with $\beta=0, \gamma=\delta=1$ the distribution becomes normal. We also see that the Lévy skewness $\beta$ is almost always close to zero with limits similar to those of the Gaussian distribution. Examination of data files show that the scenarios closest to Gaussian are those with the perfect model and a limited magnitude of disturbances (or none). On the other hand, the smallest values of the stability factor happen for scenarios with models farthest from ideal ones.

We also see that the $k$ factor of the GEV distribution does not vary much and thus it is the least informative and may be skipped from further investigation. The effect of Johnson $S_{U}$ shaping factors is unclear.

Analysis of data included in Table 6 shows that selected parameters are not cross correlated significantly and as such can be still considered potential candidates for loop quality assessment, except of the $k$ factor of the GEV. 
Table 1. Position factors for control error distributions.

\begin{tabular}{r|r|r|r|r|r|r|r|r|}
\cline { 2 - 9 } \multicolumn{1}{c|}{} & G. $x_{o}$ & C. $\delta$ & Lev. $\delta$ & Lap. $\mu$ & SU. $\xi$ & mean & GEV. $\mu$ & mean $_{G E V}$ \\
\hline \hline average & -0.0018 & 0.0001 & 0.0008 & -0.0018 & -0.1918 & -0.1874 & -0.1560 & 0.0193 \\
min & -0.0208 & -0.0099 & -0.0353 & -0.0208 & -2.1434 & -1.7752 & -0.1955 & -0.0176 \\
max & 0.0017 & 0.0026 & 0.0750 & 0.0017 & 0.0640 & 0.0007 & -0.1334 & 0.0299 \\
variance & 0.0000 & 0.0000 & 0.0001 & 0.0000 & 0.3005 & 0.2239 & 0.0002 & 0.0000 \\
\hline
\end{tabular}

Table 2. Correlations between position factors for control error distributions.

\begin{tabular}{|r|r|r|r|r|r|r|}
\cline { 2 - 7 } & G. $x_{o}$ & C. $\delta$ & Lev. $\delta$ & Lap. $\mu$ & GEV. $\mu$ & mean $_{G E V}$ \\
\hline \hline G. $x_{o}$ & $\mathbf{1}$ & & & & & \\
C. $\delta$ & $\mathbf{0 . 8 4 3 0}$ & $\mathbf{1}$ & & & & \\
Lev. $\delta$ & 0.2902 & 0.2745 & $\mathbf{1}$ & & & \\
Lap. $\mu$ & $\mathbf{1}$ & $\mathbf{0 . 8 4 3 0}$ & 0.2902 & $\mathbf{1}$ & & \\
\hline GEV. $\mu$ & 0.5867 & 0.4051 & 0.1255 & 0.5867 & $\mathbf{1}$ & \\
mean $_{G E V}$ & $\mathbf{0 . 8 2 4 4}$ & 0.6489 & 0.1659 & $\mathbf{0 . 8 2 4 4}$ & 0.7536 & $\mathbf{1}$ \\
\hline
\end{tabular}

Table 3. Review of scaling factors for control error distributions.

\begin{tabular}{r|c|c|c|c|c|c|c|c|}
\cline { 2 - 9 } & G. $\sigma$ & C. $\gamma$ & Lev. $\gamma$ & Lap.b & SU. $\delta$ & stdev & GEV. $\sigma$ & stdev \\
\hline \hline average \\
min & 0.3968 & 0.0653 & 0.0665 & 0.2140 & 1.7572 & 0.6737 & 0.4335 & 0.2066 \\
$\max$ & 0.3305 & 0.0010 & 0.0000 & 0.1247 & 0.0000 & 0.3305 & 0.3902 & 0.1712 \\
variance & 0.0016 & 0.2313 & 0.3064 & 0.3545 & 2.9494 & 3.3074 & 0.5239 & 0.2898 \\
& 0.0056 & 0.0069 & 0.0026 & 0.1698 & 0.5256 & 0.0008 & 0.0006 \\
\hline
\end{tabular}

Table 4. Correlations between scaling factors for control error distributions.

\begin{tabular}{|r|r|r|r|r|r|r|r|r|}
\cline { 2 - 9 } \multicolumn{1}{c|}{} & \multicolumn{1}{c|}{ G. $\sigma$} & \multicolumn{1}{c|}{ C. $\gamma$} & \multicolumn{1}{c|}{ Lev. $\gamma$} & Lap.b & \multicolumn{1}{c|}{ SU. $\delta$} & stdev $v_{S U}$ & GEV. $\sigma$ & stdev $v_{G E V}$ \\
\hline \hline G. $\sigma$ & 1 & & & & & & & \\
C. $\gamma$ & 0.3652 & 1 & & & & & & \\
Lev. $\gamma$ & 0.3523 & $\mathbf{0 . 9 9 2 3}$ & 1 & & & & & \\
Lap.b & 0.7151 & $\mathbf{0 . 9 0 5 7}$ & $\mathbf{0 . 8 9 3 4}$ & 1 & & & & \\
\hline SU. $\delta$ & -0.0344 & 0.0541 & -0.0034 & 0.0470 & 1 & & & \\
stdev $v_{S U}$ & 0.5998 & 0.6237 & 0.5951 & 0.7536 & -0.2670 & 1 & & \\
\hline GEV. $\sigma$ & $\mathbf{0 . 9 6 1 8}$ & 0.1373 & 0.1208 & 0.5159 & -0.0370 & 0.4536 & 1 & \\
stdev $_{G E V}$ & $\mathbf{0 . 9 4 9 3}$ & 0.1230 & 0.0996 & 0.4979 & -0.0080 & 0.4595 & $\mathbf{0 . 9 9 6 8}$ & \\
\hline
\end{tabular}

Table 5. Review of shape factors for control error distributions.

\begin{tabular}{r|r|r|r|r|r|r|r|r|}
\cline { 2 - 9 } & G.kurtosis & G.skewness & Lev. $\alpha$ & Lev. $\beta$ & Lap.b & SU. $\gamma$ & SU. $\lambda$ & GEV. $\xi$ \\
\hline \hline average & 4.9672 & -0.0703 & 0.8757 & 0.0023 & 0.2140 & 0.0398 & 0.9239 & -0.2060 \\
min & 0.3530 & -0.1136 & 0.0926 & -0.1041 & 0.1247 & -0.2515 & 0.0000 & -0.2599 \\
max & 9.8533 & 0.0426 & 1.8450 & 0.0717 & 0.3545 & 0.1508 & 4.1221 & -0.1864 \\
variance & 2.7282 & 0.0008 & 0.1982 & 0.0009 & 0.0026 & 0.0045 & 0.7554 & 0.0001 \\
\hline
\end{tabular}

Table 6. Correlations between shape factors for control error distributions.

\begin{tabular}{|r|r|r|r|r|r|r|r|}
\multicolumn{1}{c|}{} & G.kurtosis & G.skewness & \multicolumn{1}{c|}{ Lev. $\alpha$} & \multicolumn{1}{c|}{ Lev. $\beta$} & SU. $\gamma$ & SU. $\lambda$ & GEV. $\xi$ \\
\hline \hline G.kurtosis & 1 & & & & & & \\
G.skewness & -0.7153 & 1 & & & & & \\
\hline Lev. $\alpha$ & -0.5662 & 0.6183 & 1 & & & & \\
Lev. $\beta$ & 0.5723 & -0.3479 & -0.3512 & 1 & & & \\
\hline SU. $\gamma$ & 0.3360 & -0.0876 & -0.1658 & 0.3933 & 1 & & \\
SU. $\lambda$ & -0.6369 & 0.4928 & 0.3599 & -0.5063 & -0.7005 & 1 & \\
\hline GEV. $\xi$ & 0.6412 & -0.2629 & 0.0076 & 0.4207 & 0.3083 & -0.2712 & 1 \\
\hline
\end{tabular}


4.2.4. Normality tests. For all scenarios the normality hypothesis is rejected by both skewness and $\alpha$ stability tests. Here Kolmogorov-Smirnov, Anderson-Darling, Shapiro-Wilk and Lilliefors rejected the normality hypothesis in all cases. Smirnov-Cramer von Mises rejected it in 110 cases out of 162 scenarios.

Additionally, fitting indexes in the form of the mean square error between the PDF value and the histogram are calculated. This shows that Gauss, the GEV and Johnson $S_{U}$ are never best fitted. Laplace succeeded in 3 cases $(2 \%)$ with the optimal model and minimal error scenarios. Cauchy happened to be the best in $13(8 \%)$ cases (mostly scenarios without disturbances and dynamics misfitting in the GPC internal model), while Lévy won the rest 146 $(90 \%)$ scenarios. The above results confirm observations of industrial data (Domański, 2015), where the normal distribution is better only in approximately $\approx 6 \%$ cases.

4.2.5. Conclusion of statistical comparisons. We may formulate initial observations:

1. There are statistical factors aiming at the distribution position. It is observed that the Johnson unbounded function $S_{U}$ is not appropriate for that task, while the GEV distribution requires additional calculation of the mean value. The Laplace mean value is exactly equal to the Gaussian. Gauss, Cauchy and Lévy give almost similar results with the smallest sensitivity on simulated scenarios.

2. The Cauchy and Lévy distributions are very close to each other from the perspective of the shape factor. Simultaneously, their $\gamma$ factors are similar to the scale parameter $b$ of the Laplace PDF.

3. GEV parameters, both $\sigma$ and the standard deviation are very close to Gaussian $\sigma$.

4. Johnson $S_{U}$ seems to reflect an unlikely feature.

5. Shaping factors reflect other aspects of data.

6. Gauss, the GEV and Johnson $S_{U}$ are not good choices as we consider normality testing and distribution fitting to histograms.

7. A further analysis will aim at dynamical properties of the responses and thus the following distribution parameters will be further considered: Gauss $\sigma$, Cauchy $\gamma$, Lévy $\alpha$ and $\gamma$, and Laplace $b$.

\subsubsection{Comparison between PDF factors and CPA} measures. At first all the indexes are correlated with each other to see if there is any consistency. This analysis is made on all available data (see Table 7). High correlations with absolute values larger than 0.8 are highlighted.
We see that Laplace transform does not say much, as its $b$ scale parameter is highly correlated with both Cauchy and Lévy. Additionally both the Harris indexes $H_{\text {index }}$ and $H_{\text {index }}$ are of different origins. Classical integral measures, such as the AMP, IAE and MISE, are cross correlated with each other, but not with statistical factors. We see that the PDF scale and shape parameters of non-Gaussian functions are closely coupled. Additionally, the Lévy stability parameter $\alpha$ seems to keep similar information (tail fatness versus distribution slenderness).

4.2.7. H0: The impact of disturbances. One of the main features of a perfect loop quality assessment should be the ability to detect and identify controller tuning goodness despite any disturbances that might obscure investigation. From the perspective of the controller, the value of measure should be invariant. We are considering 13 scenarios of different disturbances (see Table 8).

The analysis starts with a comparison of the mean and variance between undisturbed and disturbed cases. Six simulations are considered: the optimal GPC model with horizons equal to 12 and 25 , the internal model with too small gain $K=1.6$ for both horizons. Analogously, simulations are run with too large gain $K=2.4$ for both the horizons.

The disturbance effect on the normal standard deviation $(\sigma)$ is presented in Fig. 6 It seems that the standard deviation is not a perfect choice. There is clear distinction between the parameters and robust measure evaluation for a better controller (larger horizon). However, we see significant variations impacted by disturbances for a too short horizon.

Next, Fig. 7 shows the same diagram for the Cauchy distribution scaling. We see an opposite situation. For a longer horizon (theoretically better GPC), the detection is poorer than in the short horizon case, when measure shows better performance and is not violated by disturbances.

Two other figures present the disturbance impact on two $\alpha$-stable factors, i.e., $\alpha$ (Fig. 8) and $\gamma$ (Fig. 9). They present similar features to the Cauchy distribution. A very clear separation is visible for the worse tuning of the controller despite disturbances. Conversely, GPC regulation with the optimal model is not well detected.

A similar analysis is sketched for the Harris index (Fig. 10), the AMP (Fig. 11), IAE (Fig. 12) and MISE (Fig. 13). It is very clear that the Harris indexes and the AMP are completely not robust against disturbances. The Harris histogram is highly and randomly scattered. The only visible pattern is that for each disturbance scenario the index has the highest value for optimal gain. On the contrary, the AMP shows a clear effect similar to $\alpha$ behavior. The histogram is grouped according to the value of the PDF stability parameter. It is strongly dependent 
Table 7. Correlation table for PDF measures and CPA indexes.

\begin{tabular}{|c|c|c|c|c|c|c|c|c|c|c|}
\hline & G. $\sigma$ & C. $\gamma$ & Lev. $\alpha$ & Lev. $\gamma$ & Lap.b & $H_{\text {index }}$ & $N_{\text {index }}$ & AMP & IAE & MISE \\
\hline$\overline{\overline{\text { G. }} \sigma}$ & $\overline{\overline{1}}$ & & & & & & & & & \\
\hline C. $\gamma$ & 0.3485 & 1 & & & & & & & & \\
\hline Lev. $\alpha$ & 0.1100 & 0.8608 & 1 & & & & & & & \\
\hline Lev. $\gamma$ & 0.3373 & 0.9924 & 0.8813 & 1 & & & & & & \\
\hline Lap.b & 0.7022 & 0.9059 & 0.6902 & 0.8943 & 1 & & & & & \\
\hline$H_{\text {index }}$ & 0.2077 & 0.2253 & 0.1302 & 0.2563 & 0.2632 & 1 & & & & \\
\hline$N_{\text {index }}$ & -0.0291 & -0.0102 & -0.1375 & -0.0071 & -0.0228 & 0.2221 & 1 & & & \\
\hline AMP & 0.1866 & -0.3234 & -0.3144 & -0.3229 & -0.1842 & -0.1312 & 0.2534 & 1 & & \\
\hline IAE & 0.2106 & -0.2667 & -0.2625 & -0.2614 & -0.1339 & -0.0979 & 0.2477 & 0.9918 & 1 & \\
\hline MISE & 0.2920 & -0.2870 & -0.3042 & -0.2836 & -0.1122 & -0.0857 & 0.2342 & 0.9886 & 0.9938 & 1 \\
\hline
\end{tabular}

Table 8. Scenarios for disturbance invariance investigation.

\begin{tabular}{|c|c|c|}
\hline DistScen & $z(t)$ & $d(t)$ \\
\hline \hline$D 0$ & - & - \\
$D$ & $\alpha=2.00$, Ampl = average & - \\
$D 2$ & $\alpha=1.50$, Ampl = average & - \\
$D 4$ & $\alpha=1.75$, Ampl = average & - \\
$D 5$ & $\alpha=2.00$, Ampl = large & - \\
$D 6$ & $\alpha=1.50$, Ampl = large & - \\
$D 7$ & $\alpha=1.75$, Ampl = large & - \\
\hline$D 8$ & - & $\alpha=2.00$, Ampl = average \\
$D 9$ & - & $\alpha=1.50$, Ampl = average \\
$D 10$ & - & $\alpha=1.75$, Ampl = average \\
$D 11$ & - & $\alpha=2.00$, Ampl = large \\
$D 12$ & - & $\alpha=1.50$, Ampl = large \\
$D 13$ & - & $\alpha=1.75$, Ampl = large \\
\hline
\end{tabular}

on that. Observations show that these measures are not recommended in the case of disturbances and noises.

The review of histograms for integral measures IAE and MISE shows clear consistency in the drawings. The IAE detects wrong GPC tuning in a relatively better way than the MISE, especially for the worse controller model tuning.

The analysis presented in this section verified the potential robustness of the given loop quality measures against the disturbances embedded in the loop. It seems that the parameters are mostly able to detect controller misfitting despite disturbances. However, some of them are too sensitive with a highly biased detection. Especially, Harris and AMP measures witness that effect. Thus the following indexes will be taken into consideration in the further analysis:

- statistical parameters: Gauss standard deviation $\sigma, \alpha$ and $\gamma$ of stable distribution and Cauchy's $\gamma$,

- integral indexes: the IAE and MISE.

4.2.8. H1: The effect of the set-point profile. During the evaluation of the previous results, suspicions appeared that some of them may have been biased by the shape of the set-point. In the first case the set-point is in the form of a rectangular wave with varying amplitude. There

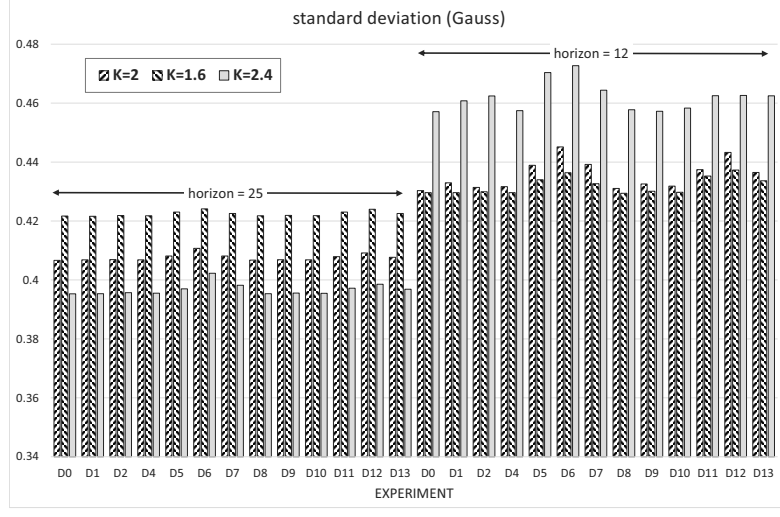

Fig. 6. Disturbance impact of the normal PDF on $\sigma$.

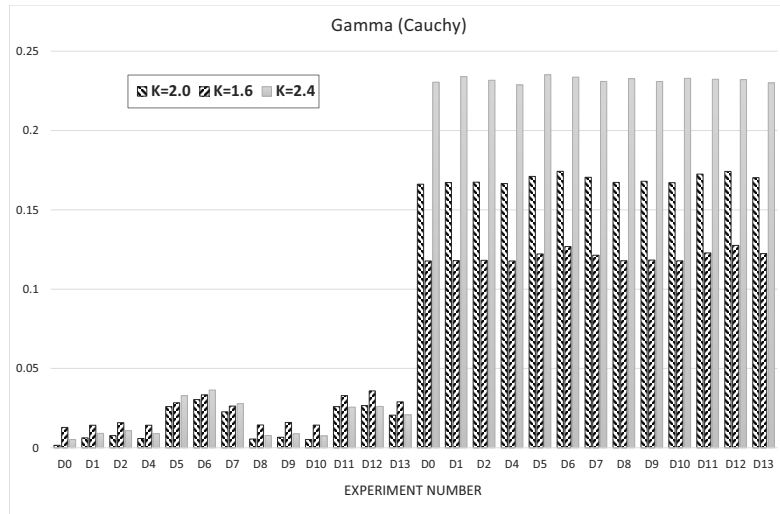

Fig. 7. Disturbance impact of the Cauchy PDF on $\gamma$.

is a second set of the same experimental results with a set-point filtered by a first order filter to exclude that effect.

Exclusion of the possible effect that a set-point character may affect results, especially the lengths of the transient period versus steady-state, is checked in the experiment below. The percentage error for each simulated measure is calculated as

$$
\Delta \eta=100 \cdot \frac{\eta^{r e c t}-\eta^{f i l t}}{\eta^{r e c t}}[\%]
$$




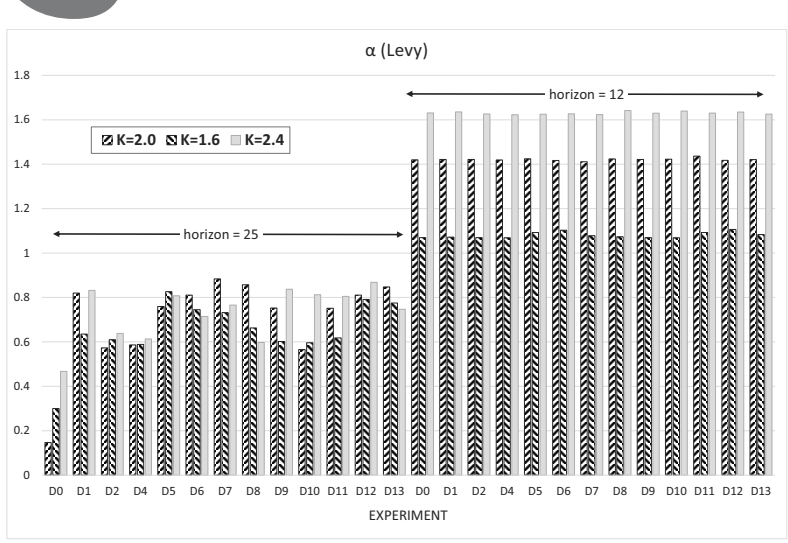

Fig. 8. Disturbance impact of the $\alpha$-stable PDF on $\alpha$.

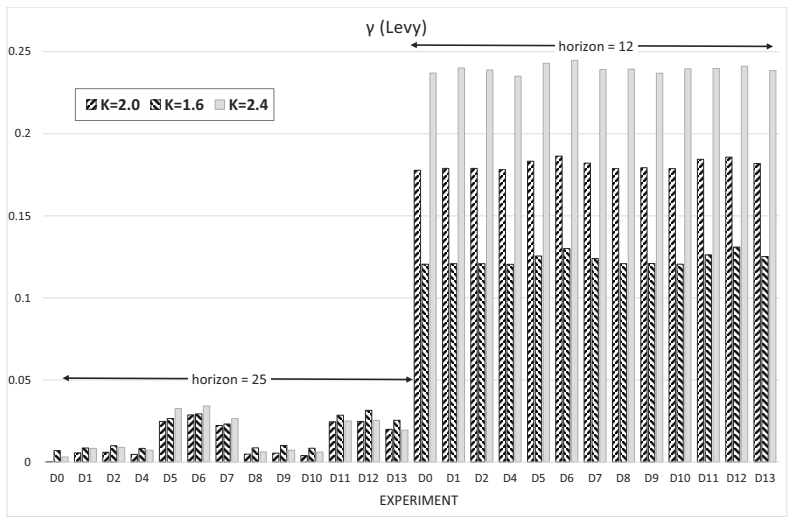

Fig. 9. Disturbance impact of the $\alpha$-stable PDF on $\gamma$.

where $\eta^{\text {rect }}$ denotes the measure for a rectangular set-point and $\eta^{f i l t}$ the same index for a filtered set-point. Next, the minimum, the maximum, the mean and the standard deviation of the measure error $\Delta \eta$ are determined for each index. Results are presented in Table 9

The comparison of the set-point effect reveals further issues. We compare the PDF range (scale) parameters, i.e., Gauss $\sigma$ and $\gamma$ of Cauchy and Lévy. We see that the error for the Gaussian standard deviation is several times larger than its counterpart for the fat-tail scalings. Even if we consider higher variability for $\gamma$, it would be safer to use a non-Gaussian distribution. The Lévy $\alpha$-stable distribution will be further analyzed as both of them give

Table 9. Measure errors statistical properties.

\begin{tabular}{|l|r|r|r|r|}
\cline { 2 - 5 } \multicolumn{1}{c|}{} & MIN & MAX & MEAN & STD.DEV \\
\hline \hline G. $\sigma$ & 8.76 & 12.10 & 10.78 & 0.73 \\
C. $\gamma$ & -6.53 & 7.04 & 1.77 & 3.31 \\
L. $\alpha$ & -39.16 & 28.40 & -3.25 & 9.47 \\
L. $\gamma$ & -10.64 & 7.62 & 1.65 & 3.47 \\
IAE & 6.09 & 12.46 & 9.98 & 1.74 \\
MISE & 16.75 & 22.73 & 20.39 & 1.31 \\
\hline
\end{tabular}

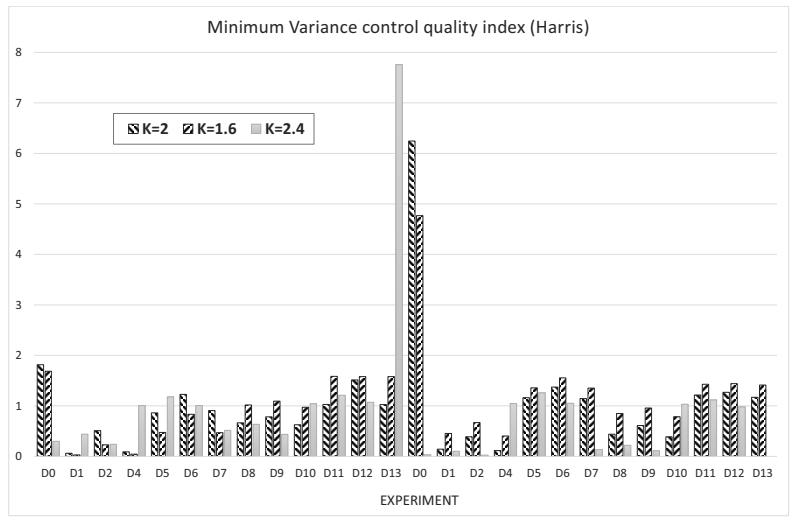

Fig. 10. Disturbance impact on the Harris performance index.

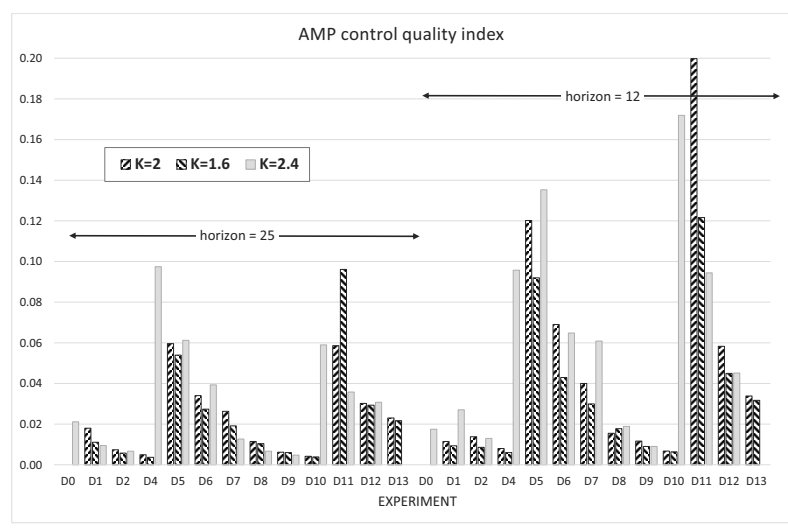

Fig. 11. Disturbance impact on the AMP performance index.

similar results.

It is additionally confirmed by the fact that a stable distribution is better fitted to the control error histograms than the other ones. Calculation of the fitting quality square error confirms it for all but one scenarios. This is a clear effect of the dominating fat tail property. We also see that the stability parameter has the largest standard deviation (variability). For safety, this will be also excluded from further investigations.

A comparison of two integral indexes favors the IAE. It has a halved mean value with a relatively similar standard deviation. Thus the MISE index will be also excluded from the further analysis. Concluding hitherto evaluations, the statistical parameters $\gamma$ of the stable distribution and the index IAE are left for examination.

4.2.9. H2: The impact of the model gain. The simulation experiments to verify the hypothesis are organized as follows. We try to answer the question if selected measures can detect a proper selection of the GPC controller embedded model gain. Nine different gain values are tested: $0.4,0.8,1.2,1.6, \mathbf{2 . 0}, 2.4,2.8,3.2,3.6$. For each of the gains three different disturbance scenarios 


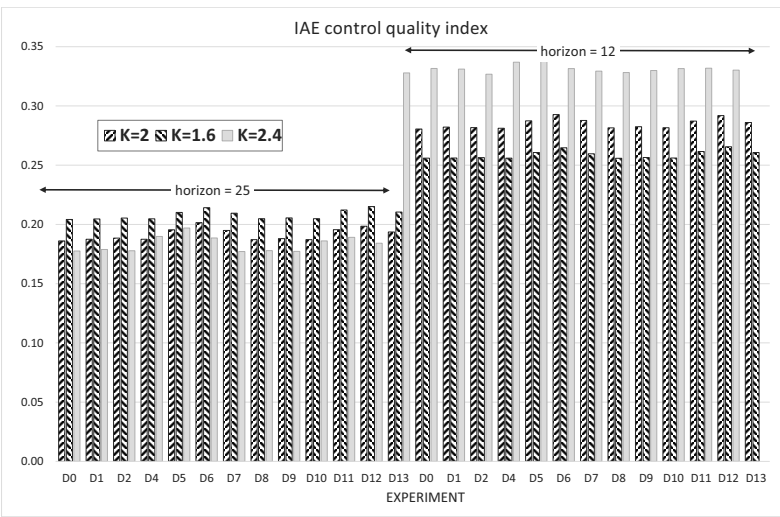

Fig. 12. Disturbance impact on the IAE performance index.

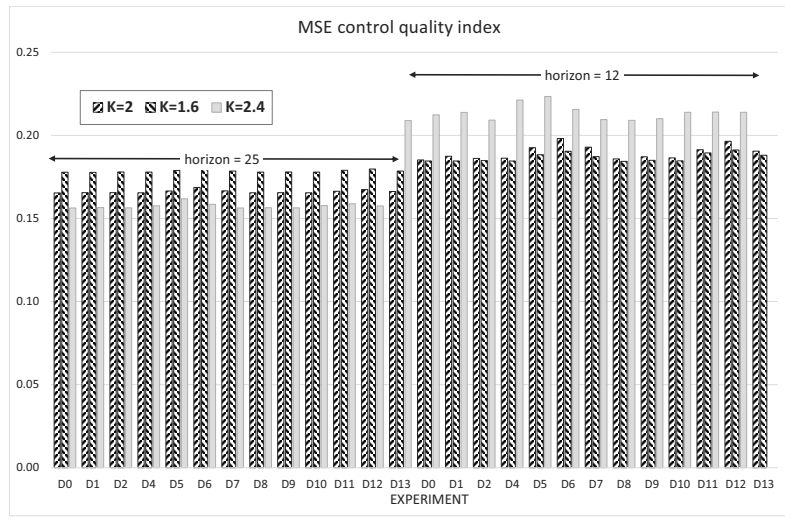

Fig. 13. Disturbance impact on the MISE performance index.

are tested: no disturbances, Gaussian noise added before the process and a $\alpha$-stable disturbance plugged after the controller.

The real value of model gain is 2.0. Thus, the plots should be able to find that value. It is very clearly seen that the curves for each of the measures have extrema indicating that point. Both $\gamma$ (Fig. 14) and the IAE (Fig. 15) correctly detect the value. All curves show good consistency. Detection of the optimal controller (the one that uses the exact model) does not depend on disturbances despite significant disturbance characteristics. The curves are almost the same.

4.2.10. H3: The impact of the model delay. A similar analysis is used to verify if selected measures can detect a proper GPC embedded model delay. Nine different delay values are tested: $4,5,6,7, \mathbf{8}, 9,10,11,12$. Three different disturbances are tested as in the previous case.

The real value of the model delay is 8.0. It is expected that plots should enable finding out this value. Unfortunately, detection is not straightforward. It works only in one case, i.e., when there are no disturbances in the loop. It is especially clearly seen for the statistical index of the stable PDF $\gamma$ (Fig. 16) and the IAE (Fig. 17). The curve for the undisturbed loop is in compliance with our expectation. First the index diminishes up to the ideal value and than starts to rise in a linear way. We may clearly point out an optimal value of the delay and additionally see that the increasing of the model delay constantly degrades control. However, simultaneously similar indexes for disturbed loops are just flat. The measures are constant independently of the model delay. It seems that loop disturbances (whatever they are) screen model delay misfitting.

We notice that a proper model delay value is hardly detected. Especially strange behavior is observed in the presence of disturbances. All the curves flatten and show nothing. This effect has to be further addressed.

4.2.11. H4: The impact of model dynamics. The same methodology is repeated to verify if selected measures can detect proper GPC embedded model dynamics. Seven values for time constant $T_{2}$ are tested: $0.5,1,5, \mathbf{1 0}, 15,20,40$ with three different disturbances as previously.

The real value of the model delay is $\mathbf{1 0}$. We may notice two different relations. For the stable PDF $\gamma$ (Fig. 18) and the IAE (Fig. 19) we see a clear ability to show the right value. Strangely, both curves have similar shapes. The IAE is more exact and $\gamma$ tends for slight overestimation.

We see that too small values of dynamics do not significantly deteriorate control quality (in the sense of the measure considered). On the contrary, for high values of $T_{2}$ the curve rapidly increases suggesting fast degradation of control quality. This behavior indicates that underestimated dynamics is not that dangerous for GPC control in contrast to the opposite case. We also observe that detection for disturbed loops is better than for the undisturbed one. This is in contrast to the model delay

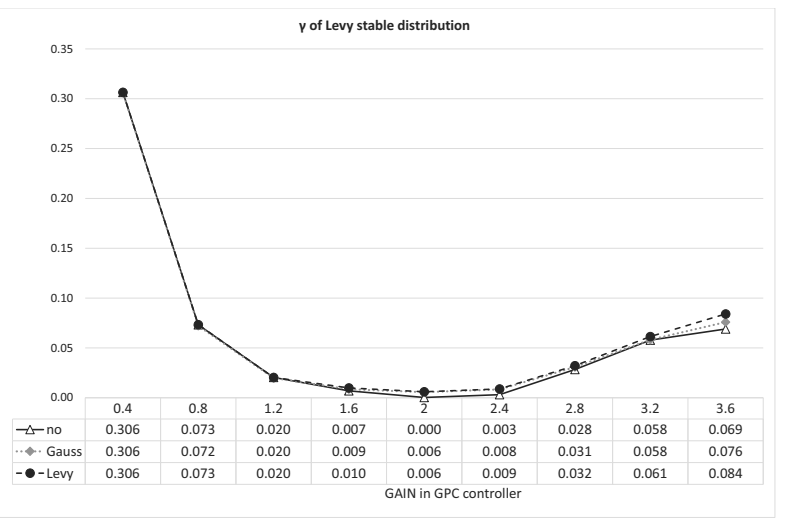

Fig. 14. Dependence of the Lévy distribution $\gamma$ on the GPC embedded model gain. 


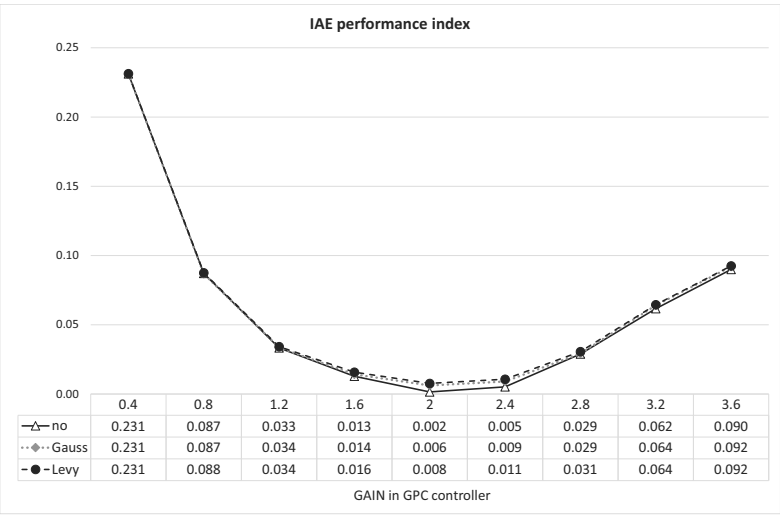

Fig. 15. Dependence of the IAE on the GPC embedded model gain.

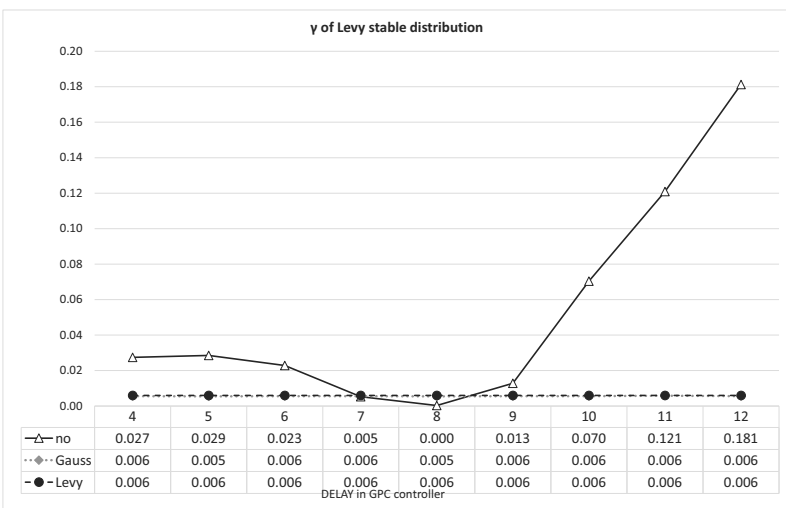

Fig. 16. Dependence of the Lévy distribution $\gamma$ on the GPC embedded model delay.

impact case. It seems that more excited signals enable dynamics misfit effect exposure.

\subsubsection{H5: The impact of the GPC prediction} horizon. Finally, similar analysis is used to check the proper GPC prediction horizon detection. Seven different horizons are tested: $10,12,15, \mathbf{2 0}, 25,30,35$ with three different applied disturbances (as previously).

In this case different results are expected. Theoretical speculations about the predictive controller clearly say that the horizon should not be too short against the process delay and the dominant time constant. On the contrary, too large horizons will only result in larger calculation efforts.

In the figures for $\gamma$ (Fig. 20) and the IAE (Fig. 21) we explicitly see that effect. The curve rapidly decreases up to a value of $\sim 20$ and after that it saturates. This means that there is no reason to increase the GPC horizon above that. We are unable to improve control performance behind this value. This effect is detected despite loop disturbances. A proper horizon value is identified in all cases.

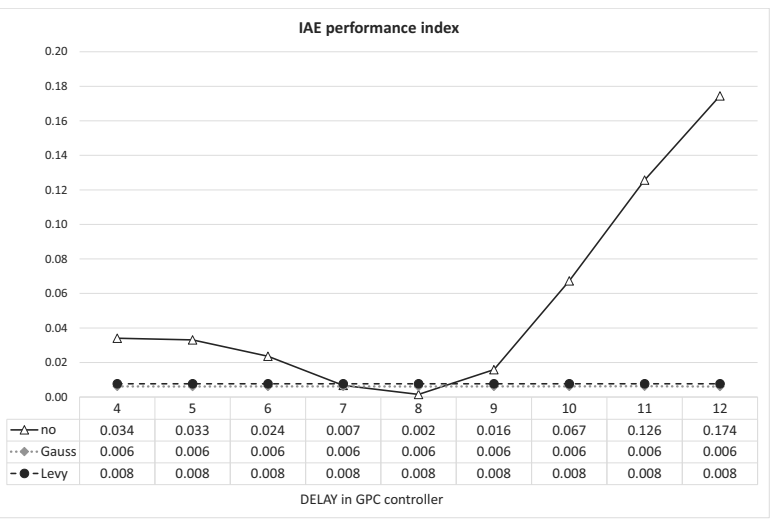

Fig. 17. Dependence of the IAE on the GPC embedded model delay.

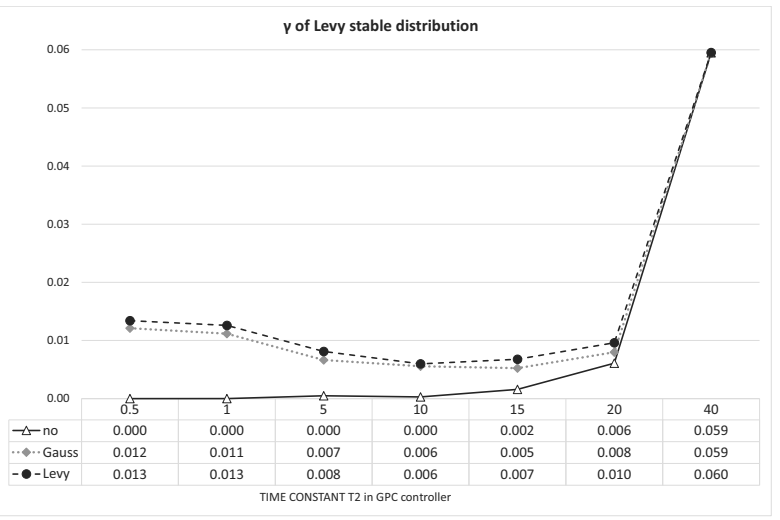

Fig. 18. Dependence of the Lévy distribution $\gamma$ on the GPC embedded model time constant $T_{2}$.

\section{Conclusions and further research}

This paper presents research on alternative, statistical control performance assessment measures used to identify the control quality of a SISO loop with the GPC controller. The analysis has been based on simulation results. All measures considered have been calculated using the control error variable. It is the best loop signal available for the analysis. First, its optimal value is zero, so any non-zero mean value at once indicates steady-state error. Any skewness clearly suggests asymmetric control, possibly due to the process nonlinearities or constraints. Finally, it should not be subject to any external trends unlike the process output and no detrending is required.

Various measures have been and analyzed to evaluate these, which are invariant to the loop external environment (disturbances, set-point, etc.). This analysis selected two robust indexes: the scaling parameter of the $\alpha$-stable distribution and the integral of the absolute error index. They are used to verify the ability of the GPC tuning quality detection. The following controller parameters have been analyzed: model gain, delay, dynamics (time 


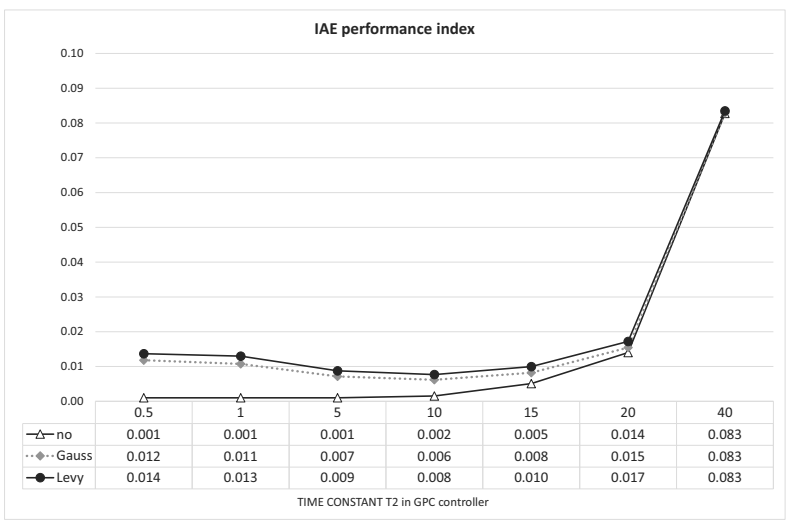

Fig. 19. Dependence of the IAE on the GPC embedded model time constant $T_{2}$.

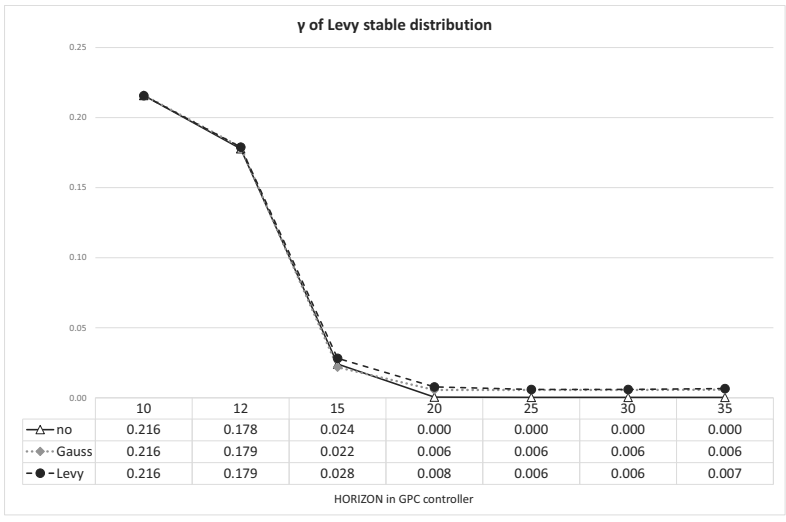

Fig. 20. Dependence of the Lévy distribution $\gamma$ on the GPC horizon.

constant) and controller prediction horizon. Comparison of the obtained results allows us to present following conclusions:

(a) Minimum variance indexes (standard and normalized) fail as their assessment effectiveness is strongly influenced by loop disturbances.

(b) Verification of the statistical properties of the control error has shown that though the normal approach is invariant to disturbances, it is biased by the character of the set-point signal. The same reason caused rejection of the stability parameter of the stable distribution function. Further analysis showed that scale factor of the $\alpha$-stable distribution seems to be the most robust. It is also proven through the control error histogram. It is strongly fat-tailed, so the Gaussian distribution fails to catch its characteristics.

(c) Standard indexes have also been evaluated. The amplitude index failed due to its strong dependence on loop disturbances. The mean square error and the absolute error indexes are compared. Following

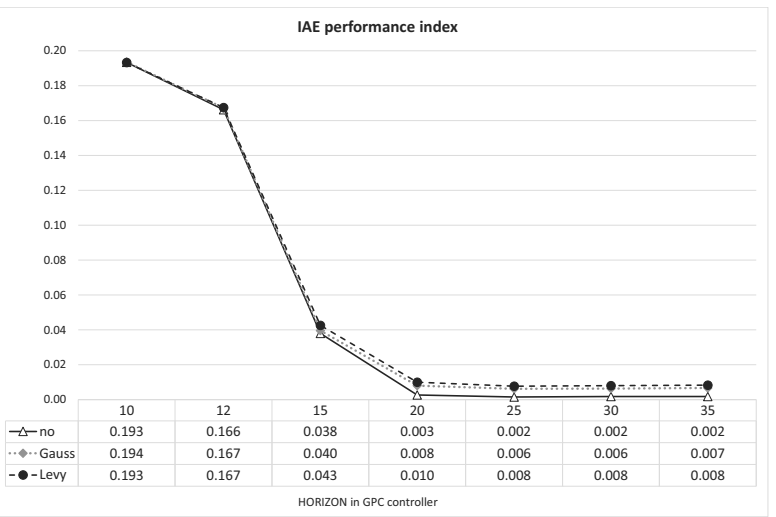

Fig. 21. Dependence of the IAE on the GPC horizon.

to literature suggestions, it is shown that the absolute error index is less invariant to the set-point characteristics.

(d) Two of selected indexes proved their detection abilities. The scaling factor and the integral of the absolute error index are able to detect model gain and dynamics misfit together with the GPC horizon selection. They only had problems with the model delay. In that case any loop disturbance is screening detection.

(e) The detection ability fails with the model delay misfit. It worked only in the ideal case of no disturbances. Any kind of disturbances interferes with identification and produces flat curves. This subject requires a detailed analysis.

The proposed approach offers new effective tools that can be used by practitioners during realization of industrial projects. The exchange of the standard deviation with the scaling factor is an easy way to improve the assessment of control loops equipped with predictive controllers.

The presented analysis opens new areas for further investigation. The problem in delay misfit detection should be reviewed. Statistical properties of the control error signal also require attention. This analysis should incorporate not only simulation, but also a review of real industrial data. Especially the aspect of long-tails needs to be addressed.

\section{References}

Anderson, T.W. and Darling, D.A. (1954). A test of goodness-of-fit, Journal of the American Statistical Association 49(268): 765-769.

Åström, K.J. (1967). Computer control of a paper machine-an application of linear stochastic control theory, IBM Journal 11(4): 389-405. 
Axensten, P. (2006). Cauchy CDF, PDF, inverse $\mathrm{CDF}$, parameter fit and random generator, http://www.mathworks.com/matlabcentral/ fileexchange/11749-cauchy/

Borak, S., Misiorek, A. and Weron, R. (2011). Models for heavy-tailed asset returns, in P. Cizek et al. (Eds.), Statistical Tools for Finance and Insurance, 2nd Edn., Springer, New York, NY, pp. 21-56.

Camacho, E.F. and Bordons, C. (1999). Model Predictive Control, Springer, London.

Choudhury, M.A.A.S., Shah, S. and Thornhill, N. (2008). Diagnosis of Process Nonlinearities and Valve Stiction, Springer, Heidelberg.

Clarke, W. and Mohtadi, C. (1989). Properties of generalized predictive control, Automatica 25(5): 859-875.

Clarke, W., Mohtadi, C. and Tuffs, P.S. (1987a). Generalized predictive control. I: The basic algorithm, Automatica 23(2): 137-148.

Clarke, W., Mohtadi, C. and Tuffs, P.S. (1987b). Generalized predictive control. II: Extensions and interpretations, Automatica 23(2): 149-160.

Cramer, H. (1928). On the composition of elementary errors, Scandinavian Actuarial Journal 1928(1): 13-74.

Cross, R. and Iqbal, A. (1995). The Rank Xerox experience: Benchmarking ten years on, in A. Rolstadås (Ed.), Benchmarking-Theory and Practice, Springer US, Boston, MA, pp. 3-10.

Domański, P.D. (2015). Non-Gaussian properties of the real industrial control error in SISO loops, Proceedings of the 19th International Conference on System Theory, Control and Computing, Cheile Gradistei, Romania, pp. 877-882.

Domański, P.D. (2016). Non-Gaussian and persistence measures for control loop quality assessment, Chaos: An Interdisciplinary Journal of Nonlinear Science 26(4): 043105.

Eisenhart, C. (2006). Laws of error. III: Later (non-Gaussian) distributions, in S. Kotz et al. (Eds.), Encyclopedia of Statistical Sciences, Wiley, Hoboken, NJ, Chapter 6.

Harris, T. (1989). Assessment of closed loop performance, Canadian Journal of Chemical Engineering 67(5): 856-861.

Hill, I.D., Hill, R. and Holder, R.L. (1976). Algorithm AS 99: Fitting Johnson curves by moments, Journal of the Royal Statistical Society C: Applied Statistics 25(2): 180-189.

Horch, A. and Isaksson, A.J. (1998). A modified index for control performance assessment, Proceedings of the 1998 American Control Conference, Philadelphia, PA, USA, Vol. 6, pp. 3430-3434.

Hugo, A.J. (2006). Performance assessment of single-loop industrial controllers, Journal of Process Control 16(8): 785-794.

Jelali, M. (2006). An overview of control performance assessment technology and industrial applications 14(5): 441-466.
Jelali, M. (2013). Control Performance Management in Industrial Automation: Assessment, Diagnosis and Improvement of Control Loop Performance, Springer-Verlag, London.

Koutrouvelis, I.A. (1980). Regression-type estimation of the parameters of stable laws, Journal of the American Statistical Association 75(372): 918-928.

Ławryńczuk, M. (2015). Nonlinear state-space predictive control with on-line linearisation and state estimation, International Journal of Applied Mathematics and Computer Science 25(4): 833-847, DOI: 10.1515/amcs-2015-0060.

Lilliefors, H. (1967). On the Kolmogorov-Smirnov test for normality with mean and variance unknown, Journal of the American Statistical Association 62(318): 399-402.

Maciejowski, J.M. (2002). Predictive Control with Constraints, Prentice Hall, Harlow.

Ordys, A., Uduehi, D. and Johnson, M.A. (2007). Process Control Performance Assessment-From Theory to Implementation, Springer, London.

Paulonis, M.A. and Cox, J.W. (2003). A practical approach for large-scale controller performance assessment, diagnosis, and improvement, Journal of Process Control 13(2): 155-168.

Schäfer, J. and Cinar, A. (2004). Multivariable MPC system performance assessment, monitoring, and diagnosis, Journal of Process Control 14(2): 113-129.

Seborg, D.E., Mellichamp, D.A., Edgar, T.F. and Doyle, F.J. (2010). Process Dynamics and Control, Wiley, Hoboken, NJ.

Shapiro, S.S. and Wilk, M.B. (1965). An analysis of variance test for normality (complete samples), Biometrika 52(3-4): 591-611.

Shinskey, F.G. (2002). Process control: As taught vs. as practiced, Industrial and Engineering Chemistry Research 41(16): 3745-3750.

Smuts, J.F. and Hussey, A. (2011). Requirements for successfully implementing and sustaining advanced control applications, ISA POWID Conference, Research Triangle Park, NC, USA, pp. 89-105.

Spinner, T., Srinivasan, B. and Rengaswamy, R. (2014). Data-based automated diagnosis and iterative retuning of proportional-integral (PI) controllers, Control Engineering Practice 29: 23-41.

Srinivasan, B. and Rengaswamy, R. (2012). Automatic oscillation detection and characterization in closed-loop systems, Control Engineering Practice 20(8): 733-746.

Tatjewski, P. (2007). Advanced Control of Industrial Processes, Structures and Algorithms, Springer, London.

Tatjewski, P. (2010). Supervisory predictive control and on-line set-point optimization, International Journal of Applied Mathematics and Computer Science 20(3): 483-495, DOI: 10.2478/v10006-010-0035-1.

Tatjewski, P. (2014). Disturbance modeling and state estimation for offset-free predictive control with state-space process models, International Journal of Applied Mathematics and Computer Science 24(2): 313-323, DOI: 10.2478/amcs-2014-0023. 
Thornhill, N.F. and Shah, S.L. (2005). New directions in control loop assessment and diagnosis, Computing \& Control Engineering Journal 16(4): 18-22.

Yang, X. and Maciejowski, J.M. (2015). Fault tolerant control using Gaussian processes and model predictive control, International Journal of Applied Mathematics and Computer Science 25(1): 133-148, DOI: 10.1515/amcs-2015-0010.

Zhuo, H. (2009). Research of performance assessment and monitoring for multivariate model predictive control system, 4th International Conference on Computer Science \& Education, Nanning, China, pp. 509-514.

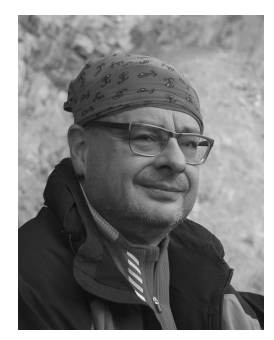

Paweł D. Domański was born in Warsaw, Poland, in 1967. He received his MSc in 1991 and his $\mathrm{PhD}$ in 1996, both in automatic control from the Warsaw University of Technology, Faculty of Electronics and Information Technology. $\mathrm{He}$ has been working at the Institute of Control and Computational Engineering of that university since 1991. Apart from scientific research, he has participated in dozens of industrial implementations of APC and optimization in power and chemical industry. He is the author of more than 80 publications in books, journals and conferences. His main research interest is in industrial APC applications, control performance quality assessment and optimization.

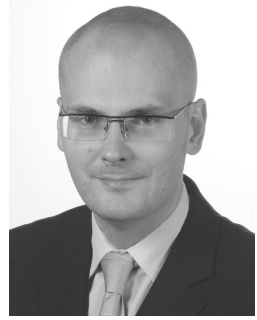

Maciej Lawryńczuk was born in Warsaw, Poland, in 1972. He received his MSc in 1998 his $\mathrm{PhD}$ in 2003, and his DSc in 2013, all in automatic control from the Warsaw University of Technology, Faculty of Electronics and Information Technology. Since 2003 he has been employed at the same university at the Institute of Control and Computation Engineering: in 20032004 as a teaching assistant, in $2004-2015$ as an assistant professor and since 2015 as an associate professor. He is the author or a co-author of 6 books and more than 100 other publications, including 30 journal articles. His research interests include advanced control algorithms, in particular, MPC algorithms, setpoint optimization algorithms, and soft computing methods, especially neural networks, modeling and simulation.

Received: 11 May 2016

Revised: 2 September 2016

Re-revised: 30 September 2016

Accepted: 12 January 2017 